\title{
Total extraperitoneal hernia repair and its associated pitfalls
}

\author{
Nasra Alam ${ }^{1}$, Aali J. Sheen ${ }^{1,2}$ \\ ${ }^{1}$ Academic Department of Hernia Surgery, Manchester University NHS Foundation Trust, Manchester M13 9WL, UK. \\ ${ }^{2}$ Department of Groin Surgery, Fortius Clinic, London W1H 6EQ, UK.
}

Correspondence to: Prof. Aali J. Sheen, Department of Academic Hernia Surgery, Manchester University, NHS Foundation Trust, Manchester M13 9WL, UK. E-mail: Aali.sheen@mft.nhs.uk

How to cite this article: Alam N, Sheen AJ. Total extraperitoneal hernia repair and its associated pitfalls. Mini-invasive Surg 2021;5:48. https://dx.doi.org/10.20517/2574-1225.2021.65

Received: 11 Mar 2021 First Decision: 15 Jun 2021 Revised: 16 Jun 2021 Accepted: 16 Jun 2021 Published: 15 Oct 2021

Academic Editors: William W. Hope, Giulio Belli Copy Editor: Xi-Jun Chen Production Editor: Xi-Jun Chen

\begin{abstract}
Minimally invasive surgery over the last three decades has provided a credible alternative for the treatment of inguinal hernias. One of the main techniques involved utilises the creation of an extraperitoneal space, thereby avoiding the need to enter the abdominal cavity. The totally extraperitoneal (TEP) inguinal hernia repair is described as well as the common and more serious complications that are possible. TEP has a proven track record of expertise for the surgical treatment of inguinal hernias, but has a steeper learning curve, with more serious complications such as vascular and bladder injuries, which are explored in more detail. The key to managing any such serious complications is early recognition. Rectus sheath hematomas secondary to inferior epigastric artery injury usually require only conservative measures such as close observation with the requirement for any embolization of any arterial bleed a rare event. Bladder injuries if recognized at the time of surgery require immediate repair, with late presentation inevitably needing more invasive intervention for a potentially septic patient. TEP remains an excellent repair with caveats of serious complications which are rare at $<0.5 \%$ however, they must be discussed and be part of the consent process prior to any repair taking place.
\end{abstract}

Keywords: TEP inguinal hernia, complications of TEP, bladder injuries, inferior epigastric artery injury, rectus sheath hematoma, bruising, chronic pain 


\section{INTRODUCTION}

Inguinal hernias are a common problem worldwide and have a prevalence of $1.7 \%$ in the adult population. This rises to $4 \%$ in patients aged over 45 years as their incidence increases with age and they constitute $75 \%$ of all abdominal wall hernias ${ }^{[1]}$. It affects men more commonly than women (lifetime risk $27 \%$ in men and $3 \%$ in women $)^{[1,2]}$. Surgical management is advised to reduce the complications associated with inguinal hernias including strangulation and subsequent bowel obstruction. Hernia repairs were commonly carried out using Lichtenstein or plug repairs but with the advent of minimally invasive techniques, laparoscopic repairs are becoming more common, more so, over the last two decades. In 2015, the Swedish Hernia Registry reported that $28 \%$ of inguinal hernias were repaired using minimally invasive surgery and $64 \%$ were using a Lichtenstein hernioplasty ${ }^{[3]}$.

Several studies have demonstrated the advantages of laparoscopic repair over conventional open repair techniques, including reduced post-operative pain and a shorter recovery time ${ }^{[4-6]}$. The abdomen can also be examined for other unsuspected hernias, such as femoral hernias in women. However, there is an associated longer learning curve and a greater risk of intra-operative complications with the laparoscopic approach ${ }^{[7]}$. Laparoscopic repair is more commonly used for the repair of bilateral inguinal hernias and recurrent hernias, as well as recently increasingly for some specialised hernias such as a sportsman's hernia ${ }^{[8]}$.

The two widely used laparoscopic techniques are trans-abdominal peritoneal repair (TAPP) and totally extraperitoneal (TEP) repair. Despite some clear advantages over open repair, laparoscopic techniques have associated complications, which are not seen with traditional open repairs ${ }^{[\rho]}$. The recognised learning curve for both minimal access methods invariable remains steeper, with TAPP shown to be marginal as quicker to learn than TEP, due to the latter's increased dissection in the extraperitoneal plane which is required, but both have the risk of visceral \& vascular injury, albeit small. But in TAPP as the peritoneal cavity is entered it has the additional risks of adhesions, small bowel injury as well as the risk of port-site hernias over TEP $(0.27 \% v \text { s. } 0.1 \% \text { for TEP })^{[10-12]}$. Some reports suggest that for TEP the learning curve is between 50 and 100 cases to gain full proficiency of the operation as well as the ability to deal with complications ${ }^{[13]}$. In this review, we will focus on TEP repair and its complications.

\section{TECHNIQUE}

TEP hernia repair involves a transverse incision lateral to the umbilicus, followed by identification and an incision of the nterior rectus sheath to identify the rectus muscle, which is then retracted to expose the posterior rectus sheath on the affected side. Above the posterior rectus sheath, the retromuscular/preperitoneal space developed and entered using a balloon trocar or blunt port, confirming its position with the camera ${ }^{[8]}$. The balloon trocar is moved laterally, back and forth, opening up the Retzius space and Bogros' space laterally. Once the pre-pneumoperitoneum has been established, two $5 \mathrm{~mm}$ ports are inserted in the midline above the pubic symphysis and blunt dissection is carried out from the midline, lateral and below the inferior epigastric vessels and to the level of the anterior superior iliac spine to the pubic bone inferiorly (cooper's ligament). The triangle of doom is exposed by retracting the peritoneum, the hernia sac dissected off the cord structures, and the vas deferens and testicular vessels are elevated. The indirect hernia sac and any significant cord lipoma are reduced and there should be a wide view of the pubic tubercle, and the insertions of the conjoined tendon and rectus muscles ${ }^{[7,8]}$. A polypropylene or other synthetic lightweight mesh is placed flat over the dissected area, above the defect to Cooper's ligament and across the midline, covering the regions of direct, indirect (myopectineal orifice), femoral and obturator hernias ${ }^{[14]}$. In a bilateral inguinal hernia repair, the same dissection is undertaken on the contralateral side with another mesh placed ensuring an overlap is achieved with the first mesh in the midline over cooper's ligament. Methods for fixation include metal tacks, absorbable tacks, no fixation, sutures, self-fixating mesh 
or tissue glue. Glue or atraumatic mesh fixation has shown to have a lower risk of complications such as nerve injury but especially both short- and long-term, chronic groin pain ${ }^{[15]}$. The pre-peritoneum is then reduced, and the skin is closed using absorbable sutures ${ }^{[7,8]}$. Patients are usually discharged the same day or the following day.

\section{COMPLICATIONS}

Immediate complications that are possible at the time of surgery include visceral injury (bowel and bladder), vascular injury, injury to the vas deferens as well as the spermatic cords. Immediately after surgery, patients can experience wound complications, bruising, scrotal swelling, seroma formation and hematomas. Delayed or late complications include adhesions (to mesh as well as adhesional bowel obstruction), fistula formation, testicular atrophy, nerve entrapment, and incisional hernia or a recurrence as well as chronic pain ${ }^{[7,14,16]}$.

With seroma formation, urinary retention can also be a problem seen post hernia repair, but this is more likely in older male patients secondary to prostatic hypertrophy. The incidence of post-operative urinary retention varies from $1 \%-3 \%$ and other risk factors as well as increasing age $>60$ years including a history of benign prostatic hypertrophy, previous bladder neck surgery and an anaesthetic time of greater than $2 \mathrm{~h}^{[16]}$. The incidence of seroma formation following laparoscopic repair is 5\%-7\% and is more common following dissection of both large indirect \& direct hernias, especially L3 \& M3 hernias (as per the EHS classification of groin hernias). Seromas will often resolve with time and do not require aspiration unless there are signs of infection or discomfort ${ }^{[16]}$.

\section{Peritoneal injuries}

Incorrect placement of the balloon trocar or simply sometimes dissection of the hernial sac may result in a breach of the peritoneum [Figure 1A], causing a pneumoperitoneum. Small peritoneal defects can be closed using a variety of methods; suturing, the use of clips [Figure 1B], or Endoloops. Closing the peritoneal defect avoids the loss of $\mathrm{CO}_{2}$ into the peritoneal cavity, and therefore allows the preperitoneal workspace to be maintained.

Larger peritoneal tears essentially may force conversion to a TAPP repair, and thus is associated with the risks of a TAPP including visceral injury, adhesions, and port site hernias ${ }^{[11]}$.

\section{Vascular injuries}

Vascular injuries can occur with laparoscopic TEP repair as the inferior epigastric artery, external iliac vessels, corona mortis as well as spermatic cord vessels are all exposed during surgery. Any vascular injury occurring during hernia surgery can often lead to conversion to an open procedure albeit this is a rare event. The inferior epigastric artery is the most frequently injured vessel, which can be damaged by balloon dissection or using the camera to create the preperitoneal space [Figure 2A]. Most bleeding can be controlled using clips or cautery. Inferior epigastric arteries can be ligated by the use of clips at the time of surgery especially if inadvertently damaged, otherwise, there is always a risk of a significant retro-rectus hematoma. If such a hematoma develops and it is not causing pain or discomfort, then simple monitoring is adequate, otherwise surgical exploration may be required especially if acute and expanding, which can involve further laparoscopy, or a laparotomy as needed. If an acute hematoma does require surgical intervention, then it is recommended that the inferior epigastric vessels are ligated as these are the most likely sources of the bleed. 

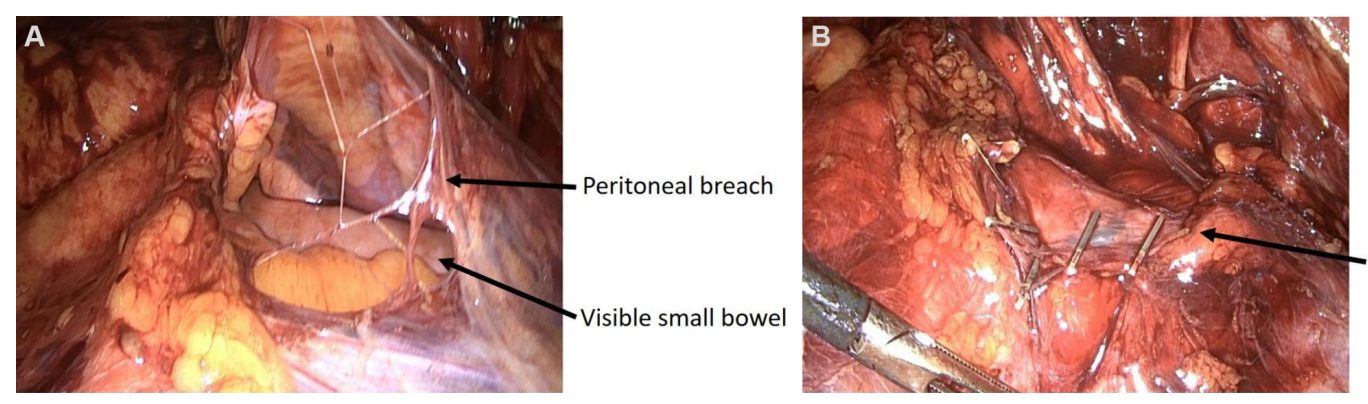

Peritoneal breach repaired by the use of $5 \mathrm{~mm}$ clips

Figure 1. Peritoneal breach showing underlying small bowel during dissection for a right inguinal TEP repair (A). Peritoneal breach repaired by the use of $5 \mathrm{~mm}$ endo-clips (B). TEP: Totally extraperitoneal.
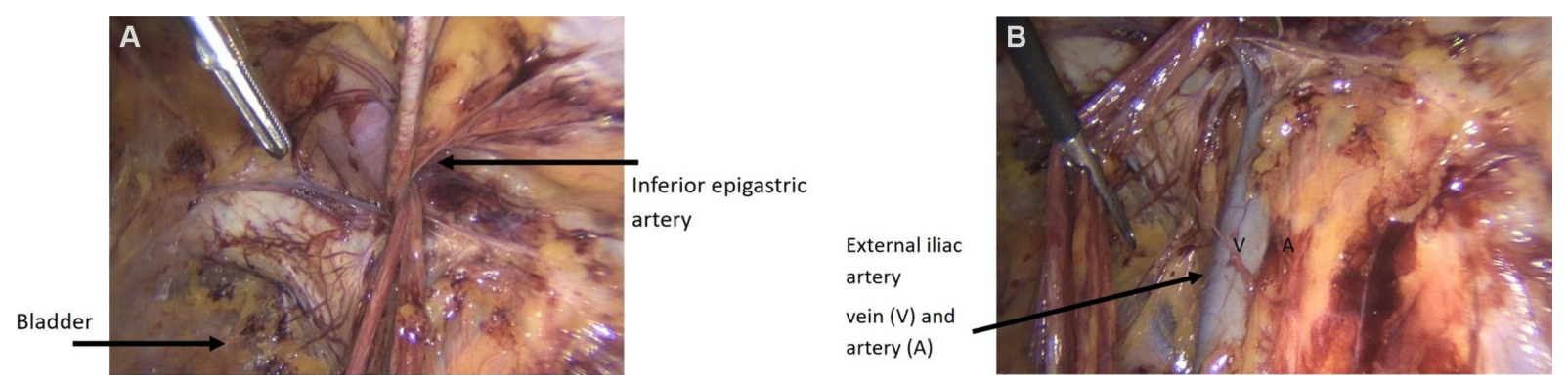

Figure 2. (A) Image showing TEP dissection and sites of possible injury with the inferior epigastric artery and the bladder. Inferior epigastric artery can cause a rectus sheath hematoma. It should be clipped if injury is inadvertently caused. (B) Image depicting the external iliac vessels, injury to these most likely will require intervention by vascular surgeons. TEP: Totally extraperitoneal.

An acutely diagnosed bleed, within the first $6 \mathrm{~h}$ of surgery, should therefore be assessed with CT imaging with contrast and any bleeding vessel can be subjected to arterial embolization to control a side branch bleed, which is largely very effective with experienced vascular radiology expertise availability.

Late presentations such as rectus sheath hematoma are usually due to a small bleed from a branch of the inferior epigastric artery or vein [Figure $2 \mathrm{~A}$ ]. These normally required no further intervention, will present with a painful rectus sheath hematoma which is diagnosed after imaging and can be managed with embolization of the vessel in the acute phase. More serious injuries will be to the external iliac vessels [Figure $2 \mathrm{~B}$ ], which will require early or immediate vascular surgical intervention.

Ischaemic orchitis can be caused by injury to the vas deferens or spermatic vessels, resulting in testicular atrophy, chronic testicular pain, or retrograde ejaculation ${ }^{[16,17]}$. It presents with painful testicular inflammation in the first 5 days post-operatively, with an incidence of $0.05 \%-0.1 \%$ in patients with large inguinoscrotal hernias. Treatment is with anti-inflammatories and scrotal suspension.

\section{Bladder injuries}

Injury to the bladder during TEP repair is rare and has a reported incidence of less than $0.5 \%$ but it lies in an anatomically vulnerable position and is easily seen during this operation lying below the ilio-pectineal (cooper's) ligament [Figure 2A] ${ }^{[18,19]}$. Bladder injuries are most likely to occur during port placement, or when dissecting a large direct hernial sac. The injury is usually recognised when urine is seen in the extraperitoneal space and it is therefore important that patients empty their bladder completely prior to surgery to reduce this risk as a full bladder will not only prevent dissection but be more prone to injury. Bladder injuries can often be repaired endoscopically using a one-layer or two-layer approach for a visible 
injury ${ }^{[19]}$ and a urinary catheter is left in situ for 5-10 days ${ }^{[16]}$. If a bladder injury is noted at the time of initial dissection, it is recommended that the repair is undertaken at the time, with the help of a Urologist if possible recommended. Surgery can of course be completed, and a check cystogram should be undertaken on at least day 10 prior to removal of the catheter. It is important to consent all patients for the risk of bladder injury prior to the operation.

Steps for managing inadvertent bladder injury at the time of TEP repair:

(1) Do not panic;

(2) Seek urology advice;

(3) Place an extra $12 \mathrm{~mm}$ port to aid in introducing 2/0 needles and suturing the bladder defect;

(4) Double layer repair with the introduction of a urinary catheter under the vision;

(5) $100 \mathrm{~mL}$ of saline used to inflate the bladder under vision to check a water-tight repair;

(6) Complete surgery and check cystogram after 10 days prior to catheter removal.

If a bladder injury is not recognised at the time of surgery patients will normally re-present early with severe lower abdominal pain, haematuria, rising inflammatory markers, sepsis with CT imaging identifying pre peritoneal fluid. It is very difficult to reattempt laparoscopic surgery, although not impossible, but a laparotomy may be inevitable with subsequent bladder repair and urinary catheterization for 10 days. Describing a spectrum of complications from TEP repairs this would be one of the more serious sequelae as well as an unrecognized bowel complication requiring a laparotomy, normally as a result of an unrecognised breach of the peritoneum, allowing small bowel to adhere to the mesh.

\section{Mesh complications (bladder)}

The use of prosthetic mesh is becoming increasingly common in hernia repairs, and therefore complications with mesh migration or mesh erosion albeit rare, have also been reported. Both TAPP and TEP hernia repairs are associated with mesh erosion into the bladder and can occur anywhere between 3 months to 10 years post-operatively ${ }^{[20]}$. Cases of mesh erosion into the bladder usually require re-operation and mesh removal, and in some cases a partial cystectomy, but this event is extremely rare and may be associated with an increased risk if previous pelvic surgery has been undertaken prior to the hernia repair.

\section{Chronic pain}

Pain that persists for longer than 3 months after surgery and is defined as "chronic post-operative inguinal pain (CPIP)". It can be categorised as bothersome moderate pain impacting daily activities lasting at least 3 months post-operatively and decreasing over time ${ }^{[6]}$. The incidence of chronic groin pain following TEP repair is much less than an open repair ${ }^{[1,2,2]}$. Mesh fixation using staples or tacks, however, increases the risk of chronic groin pain.

Patients that suffer complications are more likely to experience chronic groin pain. Although the chronic pain incidence is low, there are potential consequences with neuropraxia to the ilioinguinal, iliohypogastric nerves and even the genital branch of the genitofemoral nerve. The latter is one of the most commonly affected nerves in laparoscopic surgery as well as the lateral cutaneous nerves of the thigh. Patients present with an area of numbness just below the mid inguinal point on the thigh and should be managed with nerve pain killers and physiotherapy. The lateral cutaneous nerves of the thigh are more likely to be injured if dissection of the fascia is too close to these nerves laterally or by peeling the peritoneum too close to the lateral muscles on initial dissection. The lateral cutaneous nerves can be seen in very thin patients and should always be preserved with any subsequent damage presenting with pain on the lateral aspect of the 
affected thigh. Sometimes as a result of the use of traumatic fixation pain can also be caused in this area and if the pain occurs immediately after surgery, it is recommended that the patients are taken back to the theatre to remove the tacks. This manoeuvre does not avoid the complete effects of nerve damage, but it may abate the severity of the injury.

Nerve injuries described above do require careful evaluation by a groin specialist, nerve mapping, magnetic scan with neurography, with possible re-exploration and subsequent division of the nerves, but only after a failure of nerve painkilling agents, and desensitisation treatments including physiotherapy.

\section{Surgical emphysema}

This can occur after surgery especially with the TEP procedure. The extraperitoneal space that is created can allow gas to escape into this space further up the torso, with some patients experiencing even neck and facial pain because they have surgical emphysema, which is easily palpable. This complication does disappear eventually in time, reassurance and painkillers are all that is needed if the patient remains systemically well. Patients should be able to carry on normal bodily functions, daily activities and early follow up in the clinic after 5 to 7 days is all that is required.

\section{DISCUSSION}

Totally extraperitoneal repair for inguinal hernia remains a fast-growing procedure. It initially drove up the cost of the operation. However, with increasing expertise, experience, utilisation of cost-effective resources and most importantly its inception reducing the length of stay, recovery and return to normal activities has overall provided patients and healthcare with good benefits. The main caveat with any hernia surgery is of course the risk of complications and the TEP repair does rely on increasing expertise, with a recognised steeper learning curve ${ }^{[\rho]}$. Like any surgery, the consent process has profound implications on the direction a surgeon wishes to steer their patients in terms of which operation they will choose. Options should though always be given to patients especially with bilateral or recurrent hernia to undergo a minimally invasive technique. This has been shown in international and NICE guidelines ${ }^{[9,22]}$. Understanding the caveats in any surgical procedure are though profoundly important, especially in modern-day practice. The majority of patients will thankfully not experience any serious complications however, the more common ones associated with this repair would include umbilical wound infections, heavy bruising across the abdomen, early surgical emphysema, penile and scrotal swelling for male patients, which all generally subside within two to three weeks after the repair. Some patients will have debilitating seromas which require aspiration but again these are few and far between. Any more serious complications such as identification of vascular or bladder injuries at the time of surgery should be managed with at this time and by the utilization of specialist expertise if required. An unrecognised bladder or bowel injury will present with abdominal pain, sepsis, haematuria and rising inflammatory markers a few days after surgery. These are the most serious of risks that all patients should be advised of as part of the consent process, but a bladder injury if recognized, subsequently suture repaired with the placement of a urinary catheter should not cause undue long-term harm to a patient other than the discomfort of a urinary catheter for 10 days. Rectus sheath hematomas are managed conservatively with arterial embolization of any injury to the inferior epigastric artery rarely required but remaining a possible feasible treatment modality.

Overall, the morbidity associated with the TEP inguinal repair operation is small with only $5 \%-10 \%$ of patients experiencing a minor complication such as bruising, or wound infections, which all require reassurance and monitoring. It is only the serious complications, which surgeons should be aware of and their incidence is less than $0.5 \%$. 
Opponents of minimally invasive surgery will inevitably cite these risks with potentially serious complications as reasons not to engage in such techniques where open suture or mesh surgery is safe and just as effective. But in safe hands and adherence to a careful training module, such complications are rare with most patients recovering more quickly with an earlier as well as less painful return to normal activities and work.

\section{DECLARATIONS}

\section{Authors' contributions}

Made substantial contributions to the design, literature review and writing of this manuscript: Alam N, Sheen AJ

\section{Availability of data and materials}

Not applicable.

\section{Financial support and sponsorship}

None.

\section{Conflicts of interest}

Both authors declared that there are no conflicts of interest.

\section{Ethical approval and consent to participate}

Not applicable.

\section{Consent for publication}

Not applicable.

\section{Copyright}

(c) The Author(s) 2021.

\section{REFERENCES}

1. Öberg S, Andresen K, Rosenberg J. Etiology of inguinal hernias: a comprehensive review. Front Surg 2017;4:52. DOI PubMed PMC

2. Primatesta P, Goldacre MJ. Inguinal hernia repair: incidence of elective and emergency surgery, readmission and mortality. Int $J$ Epidemiol 1996;25:835-9. DOI PubMed

3. Gavriilidis P, Davies RJ, Wheeler J, de'Angelis N, Di Saverio S. Total extraperitoneal endoscopic hernioplasty (TEP) versus Lichtenstein hernioplasty: a systematic review by updated traditional and cumulative meta-analysis of randomised-controlled trials. Hernia 2019;23:1093-103. DOI PubMed PMC

4. Urkan M, Peker YS. TEP versus Lichtenstein, which one to choose? Rev Assoc Med Bras (1992) 2019;65:1201-7. DOI PubMed

5. Li J, Wang X, Feng X, Gu Y, Tang R. Comparison of open and laparoscopic preperitoneal repair of groin hernia. Surg Endosc 2013;27:4702-10. DOI PubMed

6. HerniaSurge Group. International guidelines for groin hernia management. Hernia 2018;22:1-165. DOI

7. Reiner MA, Bresnahan ER. Laparoscopic total extraperitoneal hernia repair outcomes. JSLS 2016;20:e2016. DOI PubMed PMC

8. Sheen AJ, Montgomery A, Simon T, Ilves I, Paajanen H. Randomized clinical trial of open suture repair versus totally extraperitoneal repair for treatment of sportsman's hernia. Br J Surg 2019;106:837-44. DOI PubMed

9. McCormack K, Scott NW, Go PM, Ross S, Grant AM; EU Hernia Trialists Collaboration. Laparoscopic techniques versus open techniques for inguinal hernia repair. Cochrane Database Syst Rev 2003:CD001785. DOI PubMed

10. Krishna A, Misra MC, Bansal VK, Kumar S, Rajeshwari S, Chabra A. Laparoscopic inguinal hernia repair: transabdominal preperitoneal (TAPP) versus totally extraperitoneal (TEP) approach: a prospective randomized controlled trial. Surg Endosc 2012;26:639-49. DOI PubMed

11. Shaikh AG, Soomro MI, Shaikh MS, Memon AA. Outcome of totally extra-peritoneal laparoscopic hernioplasty at a tertiary care hospital Larkana. J Pak Med Assoc 2013;63:850-3. PubMed

12. Köckerling F, Bittner R, Jacob DA, et al. TEP versus TAPP: comparison of the perioperative outcome in 17,587 patients with a primary unilateral inguinal hernia. Surg Endosc 2015;29:3750-60. DOI PubMed PMC

13. Simons MP, Aufenacker T, Bay-Nielsen M, et al. European Hernia Society guidelines on the treatment of inguinal hernia in adult 
patients. Hernia 2009;13:343-403. DOI PubMed PMC

14. Meyer A, Blanc P, Balique JG, et al. Laparoscopic totally extraperitoneal inguinal hernia repair: twenty-seven serious complications after 4565 consecutive operations. Rev Col Bras Cir 2013;40:32-6. DOI PubMed

15. Techapongsatorn S, Tansawet A, Kasetsermwiriya W, et al. Mesh fixation technique in totally extraperitoneal inguinal hernia repair A network meta-analysis. Surgeon 2019;17:215-24. DOI PubMed

16. Al Mahroos M, Vassiliou M. Laparoscopic totally extraperitoneal (TEP) inguinal hernia repair. In: Hope WW, Cobb WS, Adrales GL, editors. Textbook of hernia. Cham: Springer International Publishing; 2017. p. 99-107. DOI

17. Bittner R, Montgomery MA, Arregui E, et al; International Endohernia Society. Update of guidelines on laparoscopic (TAPP) and endoscopic (TEP) treatment of inguinal hernia (International Endohernia Society). Surg Endosc 2015;29:289-321. DOI PubMed PMC

18. Kocot A, Gerharz EW, Riedmiller H. Urological complications of laparoscopic inguinal hernia repair: a case series. Hernia 2011;15:583-6. DOI PubMed

19. Dalessandri KM, Bhoyrul S, Mulvihill SJ. Laparoscopic hernia repair and bladder injury. JSLS 2001;5:175-7. PubMed PMC

20. Li J, Cheng T. Mesh erosion into urinary bladder, rare condition but important to know. Hernia 2019;23:709-16. DOI PubMed

21. Grant AM, Scott NW, O'Dwyer PJ; MRC Laparoscopic Groin Hernia Trial Group. Five-year follow-up of a randomized trial to assess pain and numbness after laparoscopic or open repair of groin hernia. Br J Surg 2004;91:1570-4. DOI PubMed

22. Laparoscopic surgery for inguinal hernia repair. Available from: https://www.nice.org.uk/guidance/ta83. [Last accessed on 1 Jul 2021]. 